\title{
Stability Conditions for \\ Nonlinear Continuous Random Neural Networks
}

\author{
Jie TIAN, Juwei TAI, Xinsheng ZHANG \\ National Laboratory of Pattern Recognition \\ Institute of Automation \\ Chinese Academy of Sciences \\ Beijing 100080, P. R. of China
}

Keywords: Stability condition, random neural networks, equilibrium distribution, white noise, diffusion processes

\begin{abstract}
In this paper nonlinear conctinuous random nearal networks with asymmetric connection weights are considered. Some sufficient conditions for a given network to have a globally asymptotically stability equilibrium distribution for arbitrary input are derived. Finally, future research issues for these neuaral networks and briefly discussed.

The term neural network originally referred to a network of interconnected neurons. Today the neural network has come to mean any computing architection that consists of massing parallel inferconnection of simple "neural" processors. It is a network that performs some computional task (recognition, association, ect.) on a given pattern via interaction between a number of interconnected units having simple functions. Many types of neural networks are introduced and studied for various purpose since 1960 , especially in the last decade. Recently, K. Matsuoka deal with the following type of neural networks which he called as nonlinear continuons neural network with asymmetric connection weights. The dynamics of the network is represented by
\end{abstract}

$$
\left\{\begin{array}{l}
\tau \frac{d x_{i}(t)}{d \tau}=-x_{i}(t)+\sum_{j} \bar{w}_{i j} y_{j}(t)+s_{i} \\
y_{i}(t)=\bar{g}_{i}\left(x_{i}(t)\right)
\end{array}\right.
$$

where $x_{i}(t)$ is the variable that represents a state of unit neuron $i$ at time $t \quad(i=$ $1,2, \cdots, N) . s_{i}$ a constant input form the outside of the network, and $y_{i}(t)$ an output at time $t$. $\bar{w}_{i j}$ in the strength of connection from unit $j$ to unit $i, \tau$ is a time constant governing the rate of change of each unit's state. He obtained some stability condictions for these neural networks. In this paper, we consider the stable problem for system (1) with by white noise i.e. We deal with the following type of neural networks, which dynamics in represented by

$$
\left\{\begin{array}{l}
\tau \frac{d x_{i}(t)}{d \tau}=-x_{i}(t)+\sum_{j} \bar{w}_{i j} y_{j}(t)+s_{i}+\sum_{j} c_{i j} d B_{j}(t) \\
y_{i}(t)=\bar{g}_{i}\left(x_{i}(t)\right)
\end{array}\right.
$$

where $B_{j}(t), 1 \leq j \leq m$ are independent white noise, and $\left(c_{i j}\right)_{1 \leq i \leq N_{1 \leq j \leq m}}$ is constant matrix. We called system (2) as nonlinear continuons random neural networks with asymetric connection weights. Some stability conditions are obtained for these neural networks in this paper. In particular, when $\left(c_{i j}\right)=0$, our results are same as K.Matsuoka's. 